\title{
SUSTAINABILITY CRITERIA ASSESSMENT FOR LIFE-CYCLE PHASES OF PETROLEUM REFINERY PROJECTS BY MADM TECHNIQUE
}

\author{
Yaghob Gholipour, Hamidreza Hasheminasab, \\ Mohammad Kharrazi, Justas Streimikis
}

\section{Introduction}

There is convincing evidence to suggest that understanding the fundamental needs of human beings is essential if we are to develop strategies to transition society towards more sustainable forms of development (Hall, 2006). Besides, human needs satisfaction is likely to "make fewer demands on our environmental resources, but much greater demands on our moral resources" (Brown, 1982). As such, it is a moral obligation for governments, societies, industries, and individuals, to help fulfill human needs by enhancing health, safety, economy, and society, while preserving the environmental assets such as biodiversity and natural resources; i.e. to realize sustainable development.

Infrastructural projects are often non-profit projects, however, governments have to pursue them to improve living standards and to develop economies and societies. This is especially true for energy sector projects. However, these projects almost always have large negative influences on the environment. As a result, a comprehensive sustainability framework is crucial, whether developed by governments or other entities. This framework should cover the entire life-cycle of the project and should cater to various types of information needed by managers in their respective industries.

PRI projects supply a great share of world's demand for energy, and as such, could have great positive economic and social impacts. At the same time, they are notorious for their effects on environmental degradation and possible social harm. Because of these conflicting attributes, a fully connected sustainability assessment framework has to be developed for PRI projects, and the correlation between sustainability factors and life cycle phases and sub-phases should be clearly determined so that it can be used as a decision making (MADM) tool with wide applicability. To be consistent with MADM terminology, we will call these phases and sub-phases as 'Alternatives' henceforth.

One of the important attributes of PRI projects is the related technology and its quick development. Technology has a positive effect which is its ability to shape the world by influencing international conflicts, national politics, the distribution of wealth and power, gender equality, etc. On the other hand, technology has a negative influence as well. It is largely responsible for the degradation of the natural environment to the extent that ecosystems and wildlife have either been destroyed or put under serious stress (Carson, 1962). Another technology concern is the pace at which the technology is changing (Streeten, 2001). Governments should have the required flexibility to adapt to rapid technology changes. All in all, PRI projects are high-tech industries especially in the complementary treatments which occur in reformers and unifiers. Due to the inherent complexities that are caused by high tech equipment and processes, the full connectivity of the sustainability framework is of primary importance (Stjepcevic \& Siksnelyte, 2017).

\section{Literature Review}

There is a great deal of literature on social sustainability (Missimer et al., 2017; 2016; Silajdžić et al., 2015), environmental sustainability (Ferreira et al., 2015; Felix \& Gheewala, 2012; de Castro Hilsdorf et al., 2017; Shortall et al. 2015), economic sustainability (Ooba et al., 2015; Filipović \& Golušin, 2015), and sustainability integrated assessment 
(Stamford et al., 2014; Musango, 2011; AngelisDimakis et al., 2012; Halaby et al., 2017). Besides, developing infrastructural projects and achieving sustainable infrastructure is of paramount importance, and as such, it has been studied in many publications (Yao et al., 2011; Papajohn et al., 2016; Sierra et al., 2015). Sustainability of Oil and Gas Industry (OGI) companies and projects has been the subject of many studies as well (George et al., 2016; Silvestre \& Gimenes, 2017; Heravi et al., 2015; Neelis, 2008; Ahmad et al., 2016). Among OGI projects, PRI projects have been the subject of many sustainability studies in particular (Hadidi et al., 2016; Holmgren et al., 2008; Ba-Shammakh, 2010; Jovanovic et al., 2010; Mahmoud \& Shuhaimi, 2013). Sustainability indicators in all Triple bottom lines (TBL) have been considered in many of these studies (Hiremath et al., 2013; Shen et al., 2010; Shen et al., 2011; Chong et al., 2016).

Most of the previous studies track only one pass towards the development of sustainability indicators and this would increase the possibility of reduced accuracy of the framework and its application. In this study, a sustainability framework which was specifically developed for PRI projects (Hasheminasab et al., 2018), has been used as the basis to develop a connectivity model to enable practitioners and managers to assess the realization of sustainability factors and indicators in various life-cycle phases of petroleum refineries. To create this model, expert opinions have been utilized; therefore, this framework is based on available literature and at the same time relies on industry experts as well.

Many corporate world problems stem from the complexity of dealing with many criteria and stakeholders in the process of decision making (DM). Hence, during the past decades, a wide variety of DM techniques have been developed in Operations Research field, Multicriteria Decision Making (MCDM), by which the aforementioned problems would be simplified and solved (Ferreira et al., 2017). MCDM techniques are divided into Multi-Objective Decision Making (MODM) and Multi-Attribute Decision Making (MADM) (Zavadskas et al., 2014). MADM was considered as a multidisciplinary methodology for solving problems and is used as a decision making tool in many fields and studies (Mardani et al., 2015; Wang et al., 2014).

Life-cycle sustainability assessment has a life-long approach towards relevant issues. Therefore, it is a valuable tool for long-term decision making and strategic management (Stelzer et al., 2015). To use life-cycle concepts, various phases, i.e. cradle to refinery gate, refinery gate to product gate, and product gate to the grave, will be considered separately, and to be consistent with MADM terminology, these phases will be called 'Scenarios'. Although scenarios are qualitative in nature, MADM techniques are used to quantify them. Therefore, MADM based Scenarios will be used to model the life-cycle of PRI projects (Hashemkhani Zolfani et al., 2016).

\section{Methodology}

The methodology used in this study will comprise a chain of methodologies that will be used as represented in Tab. 1.

\subsection{Sustainability Framework}

So many frameworks are developed for sustainability assessment in order to be used for different projects and fields. Among these, a framework that was developed recently by the authors (Hasheminasab et al., 2018) for PRI projects, in particular, will be utilized in

\section{Tab. 1: Methodology map}

\begin{tabular}{c|c} 
Methodology & Phase \\
\hline Literature review & Sustainability indicator framework \\
\hline Concept mapping & Petroleum refinery life-cycle modeling \\
\hline Focus group & Indicator-refinery connectivity \\
\hline MADM based scenario & Life-cycle sustainability criteria assessment \\
\hline Case study & Real petroleum refinery \\
\hline
\end{tabular}


this study. This framework contains pillars, indicators, and factors which are going to be utilized in this study. For more information about the details of indicators and factors, the reader can refer to the aforementioned reference.

\subsection{Petroleum Refinery Life-Cycle Modeling}

A petroleum refinery is a complex, multidisciplinary facility which contains millions of components. In this study, in order to use a life-cycle model of a refinery with high accuracy and efficiency, a panel of highly experienced experts was formed from various related disciplines so that a comprehensive accurate model of a petroleum refinery with life-cycle focus could be developed. This panel comprised 5 experts, and during the Concept Mapping session, a model was developed which is presented in Fig. 1 and Tab. 2. In subsequent calculations, a petroleum refinery's processes and scenarios will be based on this model.

\section{Fig. 1: Life-cycle model of a petroleum refinery}

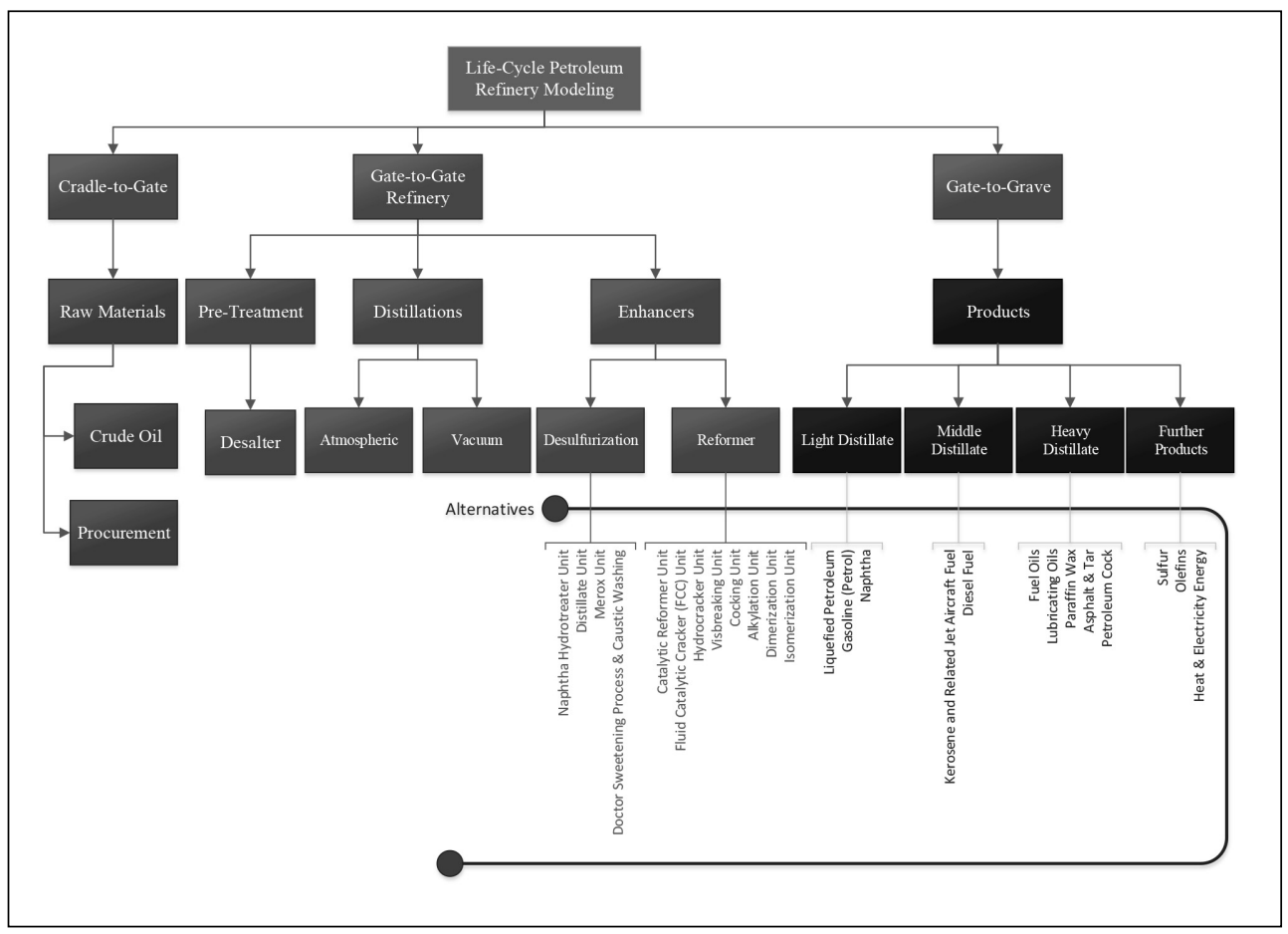

Source: own

\section{Tab. 2: Life-cycle modeling for the petroleum refinery project}

\begin{tabular}{|c|c|c|c|c|c|c|c|c|c|c|}
\hline \multirow{2}{*}{\multicolumn{2}{|c|}{$\begin{array}{c}\text { Cradle-to-Gate } \\
\text { Raw material }\end{array}$}} & \multicolumn{5}{|c|}{ Gate-to-Gate } & \multirow{2}{*}{\multicolumn{4}{|c|}{$\begin{array}{c}\text { Gate-to-Grave } \\
\text { Products }\end{array}$}} \\
\hline & & Pretreatment & Distillat & ons & Enhanc & & & & & \\
\hline Crude oil & Procurement & De-salter & Atmospheric & Vacuum & Desulfurization & Reformer & $\begin{array}{c}\text { Light } \\
\text { Distillate }\end{array}$ & $\begin{array}{c}\text { Middle } \\
\text { Distillate }\end{array}$ & $\begin{array}{c}\text { Heavy } \\
\text { Distillate }\end{array}$ & $\begin{array}{l}\text { Further } \\
\text { Products }\end{array}$ \\
\hline
\end{tabular}

Source: own 


\subsection{Calculate Connectivity}

The connectivity between indicator-based sustainability framework which was developed by the authors in a previous publication (Hasheminasab et al., 2018) and petroleum refinery life-cycle model, which was developed from the above Concept Mapping approach, was determined by a panel of experts by using a Focus Group technique. These experts had at least 15 years of related experience in their petroleum refinery-related fields. The connectivity matrix showed whether a specific sustainability factor was related to a specific alternative (according to MADM terminology). The subsequent stages of this study will only be focusing on the non-zero cells of the connectivity matrix; i.e. the zero connectivity between factors and alternatives will not be pursued any further.

\subsection{Life-Cycle Sustainability Criteria Assessment}

Based on the aforementioned steps, the problem is modeled with MADM based scenarios. In this regard, three scenarios are considered as petroleum refinery life-cycle phases (Cradle-toGate, Gate-to-Gate, and Gate-to-Grave), and the criteria are the sustainability factors. Moreover, in this decision model, every scenario has some alternatives which are evaluated through different criteria set based on the connectivity outputs. The base methodology for this stage was taken from (Hashemkhani et al., 2016) and developed and modified in accordance with the problem at hand, as follows:

\section{Step 1: Normalized decision making table}

Normalized decision matrix is calculated as follows. These equations are used if the extremum value is max or min respectively.

$$
\begin{aligned}
& \bar{x}_{i j}=\frac{x_{i j}}{\operatorname{ext}_{i}\left(x_{i j}\right)}, \forall i \in\{1 \ldots . . I\} . j \in\{1 . \ldots . J\} \text { for max case } \\
& \bar{x}_{i j}=\frac{\operatorname{ext}_{i}\left(x_{i j}\right)}{x_{i j}} . \forall i \in\{1 \ldots . . I\} . j \in\{1 . \ldots . J\} \text { for min case }
\end{aligned}
$$

\section{Step 2: Calculate WASPAS weights}

There are two types of WASPAS weights which are based on normalization and multiplication and are represented as follows respectively.

$$
\begin{aligned}
& \overline{\bar{x}}_{i j . s u m}=\bar{x}_{i j} \times W_{j} . \quad \forall i \in\{1 \ldots . I\} . j \in\{1 \ldots . . J\} \\
& \overline{\bar{x}}_{i j . m u l t}=\bar{x}_{i j} W_{j} . \quad \forall i \in\{1 \ldots . I\} . j \in\{1 \ldots . J\}
\end{aligned}
$$

\section{Step 3: weighted normalized matrix}

Final weights are calculated by the following equation:

$$
W P S_{i}=\frac{\sum_{j} \overline{\bar{x}}_{i j . s u m}+\sum_{j} \overline{\bar{x}}_{i j . \text { mult }}}{2}, \forall i \in\{1 . \ldots . I\} . j \in\{1 . \ldots . J\}
$$

\section{Step 4: Final evaluation and rankings}

The calculated weights from Step 3 are further modified in this step, based on the number of times that a criterion is repeated in various scenarios. In other words, more weight is given to criteria that are repeated more frequently, compared to the criteria that have a lower frequency:

$$
\begin{aligned}
& \overline{W_{\iota}}=\frac{W_{i} \times I_{i}}{\sum_{i}\left(W_{i} \times I_{i}\right)} \\
& \overline{A_{\iota}}=\frac{A_{i} / I_{i}}{\sum_{i}\left(A_{i} / I_{i}\right)}
\end{aligned}
$$

where $I_{i}$ represents the frequency of the $\mathrm{i}^{\text {th }}$ criteria. $W_{i}$ : primary weights that signify the absolute importance of various criteria (sustainability factors), without regard to their influence on various alternatives. $\bar{W}_{l}$ : modified weights. $A_{i}$ : primary ranks. $\bar{A}_{l}:$ modified ranks.

\section{Case Study}

A real refinery is investigated as a case study for this research. A panel of 15 experts with related experiences was selected for this study. Tab. 3 shows their competencies according to their years of experience and education level.

\begin{tabular}{l|c|c|c|c|c|c} 
& \multicolumn{3}{|c|}{ Education } & \multicolumn{3}{c}{ Experience(year) } \\
\hline & BSc. & MSc. & Ph.D. & $\mathbf{0 - 1 0}$ & $\mathbf{1 0 - 1 5}$ & Over 15 \\
\hline Number of experts & 8 & 5 & 2 & 3 & 7 & 5 \\
\hline
\end{tabular}




\begin{tabular}{c|c|c|c|c|c}
\multirow{2}{*}{$\begin{array}{c}\text { Proposed } \\
\text { Factor }\end{array}$} & \multirow{2}{*}{ Weight } & \multirow{2}{*}{ ext() } & \multicolumn{3}{|c}{ Life-Cycle Phases (Scenarios) } \\
\cline { 4 - 6 } & & & Scenario1 & Scenario2 & Scenario3 \\
\cline { 3 - 6 } & & Max/Min & A1, A2 & A3, .., A7 & A8, .., A11 \\
\hline $\mathrm{F} 1, \ldots, \mathrm{F} 101$ & $\mathrm{~W} 1, \ldots, \mathrm{W} 101$ & & & & \\
\hline
\end{tabular}

Note: F1, .., F101 are the sustainability factors (see Hasheminasab et al. (2018) for more information). A1, .., A11 are the scenarios' alternatives based on Tab. 2. W1, .., W101 are assigned weights to the absolute importance of various factors (criteria as per MADM terminology).

\section{Tab. 5: Social criteria evaluation for different scenarios}

\begin{tabular}{l|c|c|c|c|c} 
& \multicolumn{5}{|c}{ Social } \\
\hline & $C_{1}{ }^{1}$ & $C_{2}{ }$ & $C_{3}{ }^{l}$ & $C_{4}{ }^{l}$ & $C_{5}{ }^{1}$ \\
\hline Scenario1 & 2.33 & 2.71 & 2.00 & 2.20 & 1.50 \\
\hline Scenario2 & 1.83 & 1.57 & 3.20 & 2.20 & 1.67 \\
\hline Scenario3 & 2.67 & 1.29 & 2.80 & 2.00 & 0.83 \\
\hline mean & 2.28 & 1.86 & 2.67 & 2.13 & 1.33 \\
\hline Rank & 1 & 3 & 2 & 4 & 5 \\
\hline
\end{tabular}

\section{Tab. 6: Environmental criteria evaluation for different scenarios}

\begin{tabular}{l|c|c|c|c|c} 
& \multicolumn{5}{|c}{ Environmental } \\
\hline & $C_{1}{ }^{2}$ & $C_{2}{ }^{2}$ & $C_{3}{ }^{2}$ & $C_{4}{ }^{2}$ & $C_{5}{ }^{2}$ \\
\hline Scenario1 & 3.67 & 1.13 & 1.29 & 2.25 & 1.67 \\
\hline Scenario2 & 3.67 & 2.00 & 1.57 & 2.38 & 1.67 \\
\hline Scenario3 & 3.50 & 1.50 & 1.14 & 1.25 & 0.00 \\
\hline mean & 3.61 & 1.54 & 1.33 & 1.96 & 1.11 \\
\hline Rank & 1 & 3 & 4 & 2 & 5 \\
\hline
\end{tabular}

Based on the connectivity outputs, life-cycle sustainability criteria assessment is evaluated for the petroleum refinery of the case study. A decision table (Tab. 4) was created as follows and was subsequently filled by each of the aforementioned experts.

As the end result, mean value of significance of criteria are calculated, averaged across various criteria belonging to each indicator, and presented in Tabs. 5-7 for various scenarios.
To elaborate further on the steps that were taken to reach the results of Tabs. 5-7, intermediate results are partially presented in Tabs. 9-15. To be brief, in the following tables, only the first two sustainability factors are presented. Tab. 9 lists the absolute weights for the first two factors (criteria) that is the outcome of the focus group exercise. Note that, in cells that no connectivity is found (see Tab. 10) the weight is set as 0 . 


\section{Ekonomika a management}

Tab. 7: Economic criteria evaluation for different scenarios
\begin{tabular}{l|c|c|c|c|c} 
& \multicolumn{5}{|c}{ Economic } \\
\hline & $C_{1}{ }^{3}$ & $C_{2}{ }^{3}$ & $C_{3}{ }^{3}$ & $C_{4}{ }^{3}$ & $C_{5}{ }^{3}$ \\
\hline Scenario1 & 4.50 & 3.92 & 2.54 & 2.20 & 2.20 \\
\hline Scenario2 & 4.50 & 4.50 & 2.54 & 2.20 & 2.20 \\
\hline Scenario3 & 4.50 & 4.50 & 2.54 & 1.40 & 2.20 \\
\hline mean & 4.50 & 4.31 & 2.54 & 1.93 & 2.20 \\
\hline Rank & 3 & 2 & 1 & 5 & 4 \\
\hline
\end{tabular}

Source: own

Note: $C_{i}^{j}$, is the ith indicator for the jth sustainability pillar ( $\mathrm{j}=1$ for social, 2 for environmental, 3 for economical) as per Tab. 8 below. This value is computed by calculating the mean of its sustainability factor (criteria) weights.

\section{Tab. 8: Sustainability indicators}

\begin{tabular}{l|l|l}
\multicolumn{1}{c|}{ Sustainability Pillars } & \multicolumn{2}{c}{ Sustainability indicators } \\
\hline \multirow{4}{*}{ Social } & $C_{1}{ }^{1}$ & Poverty \& Equality \\
\cline { 2 - 3 } & $C_{2}{ }^{1}$ & Health \\
\cline { 2 - 3 } & $C_{3}{ }^{1}$ & Safety \& Security \\
\cline { 2 - 3 } & $C_{4}{ }^{1}$ & Education \& Training \\
\cline { 2 - 3 } & $C_{5}{ }^{1}$ & Welfare \\
\hline \multirow{5}{*}{ Environmental } & $C_{1}{ }^{2}$ & Atmosphere \\
\cline { 2 - 3 } & $C_{2}{ }^{2}$ & Water(Fresh Water, Ocean, Sea, Coast) \\
\cline { 2 - 3 } & $C_{3}{ }^{2}$ & Land \& Soil Pollution \\
\cline { 2 - 3 } & $C_{4}{ }^{2}$ & Natural Resource \\
\cline { 2 - 3 } & $C_{5}{ }^{2}$ & Biodiversity \\
\hline \multirow{5}{*}{ Economical } & $C_{1}{ }^{3}$ & Energy consumption \\
\cline { 2 - 3 } & $C_{2}{ }^{3}$ & Financial \\
\cline { 2 - 3 } & $C_{3}{ }^{3}$ & Economy Performance \\
\cline { 2 - 3 } & $C_{4}{ }^{3}$ & Occupation \\
\cline { 2 - 3 } & $C_{5}{ }^{3}$ & Earning \\
\hline
\end{tabular}

Source: own

In the next step, the connectivity between various criteria and alternatives is assessed during the Focus Group exercise. Tab. 10 shows a sample of the connectivity matrix.

In the next step, the significance of each criterion for each alternative is assessed. Tab. 11 shows the average values (taken from experts' opinions during the Focus Group exercise) for the first two factors (criteria). Where there is no connectivity, no value is provided.

Normalized values are calculated for every factor based on the related extremum values (Tab. 12).

Following the stated WASPAS methodology, the normalized weighted sum values are calculated and presented in Tab. 13. 


\section{Tab. 9: Weights and extrema definition}

\begin{tabular}{|c|c|c|c|c|c|c|c|}
\hline \multirow{2}{*}{ No. } & \multirow{2}{*}{ Pillar } & \multirow{2}{*}{ Indicator } & Factor & \multicolumn{3}{|c|}{ Weight } & \multirow{2}{*}{$\begin{array}{c}\operatorname{ext}() \\
\text { Max/Mir }\end{array}$} \\
\hline & & & Proposed Factor / EN & Sce1 & Sce2 & Sce3 & \\
\hline 1 & \multirow[b]{2}{*}{ Social } & \multirow{2}{*}{$\begin{array}{l}\text { Poverty \& } \\
\text { Equality }\end{array}$} & $\begin{array}{l}\text { Proportion of project human } \\
\text { resource living below national } \\
\text { poverty line }\end{array}$ & 5 & 0 & 5 & $\min$ \\
\hline 2 & & & $\begin{array}{l}\text { Fraction of project human } \\
\text { resource protected against } \\
\text { impoverishment by out-of- } \\
\text { pocket health expenditures }\end{array}$ & 4 & 4 & 4 & $\max$ \\
\hline
\end{tabular}

\section{Tab. 10: Connectivity measurement}

\begin{tabular}{|c|c|c|c|c|c|c|c|c|c|c|c|}
\hline \multirow[b]{3}{*}{ No. } & \multirow{2}{*}{\multicolumn{2}{|c|}{$\begin{array}{l}\text { Cradle-to-Gate } \\
\text { Raw material }\end{array}$}} & \multicolumn{5}{|c|}{ Gate-to-Gate } & \multirow{2}{*}{\multicolumn{4}{|c|}{$\begin{array}{c}\text { Gate-to-Grave } \\
\text { Products }\end{array}$}} \\
\hline & & & \multirow{2}{*}{ 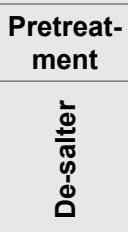 } & \multicolumn{2}{|c|}{ Distillations } & \multicolumn{2}{|c|}{ Enhancers } & & & & \\
\hline & 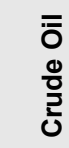 & 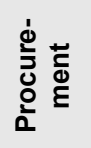 & & 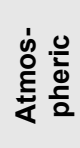 & 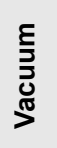 & 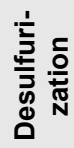 & 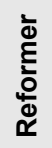 & 芯营 & $\frac{0}{\frac{0}{0}}$ & 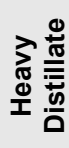 & 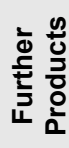 \\
\hline 1 & 1 & 1 & 0 & 0 & 0 & 0 & 0 & 1 & 1 & 1 & 1 \\
\hline 2 & 1 & 1 & 1 & 1 & 1 & 1 & 1 & 1 & 1 & 1 & 1 \\
\hline
\end{tabular}

\section{Tab. 11: Mean value of significance of each criteria for each alternative}

\begin{tabular}{|c|c|c|c|c|c|c|c|c|c|c|c|}
\hline \multirow[b]{3}{*}{ No. } & \multirow{2}{*}{\multicolumn{2}{|c|}{$\begin{array}{c}\text { Cradle-to-Gate } \\
\text { Raw material }\end{array}$}} & \multicolumn{5}{|c|}{ Gate-to-Gate } & \multirow{2}{*}{\multicolumn{4}{|c|}{$\begin{array}{c}\text { Gate-to-Grave } \\
\text { Products }\end{array}$}} \\
\hline & & & \multirow{2}{*}{ 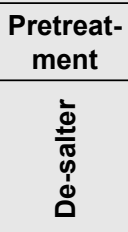 } & \multicolumn{2}{|c|}{ Distillations } & \multicolumn{2}{|c|}{ Enhancers } & & & & \\
\hline & $\begin{array}{l}\overline{0} \\
\frac{0}{0} \\
\overline{0}\end{array}$ & 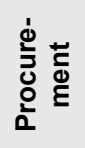 & & 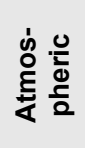 & 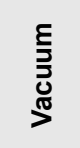 & 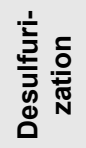 & 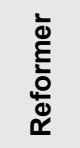 & 志永 & 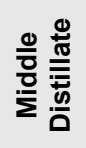 & 恋离 & 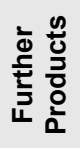 \\
\hline 1 & 1.154 & 0.615 & -- & -- & -- & -- & -- & 2.154 & 2.462 & 2.154 & 1.923 \\
\hline 2 & 2.231 & 1.154 & 1.538 & 1.769 & 1.385 & 1.538 & 1.615 & 2 & 2.231 & 2.308 & 1.769 \\
\hline
\end{tabular}




\section{Ekonomika a management}

\section{Tab. 12: Normalized values}

\begin{tabular}{|c|c|c|c|c|c|c|c|c|c|c|c|}
\hline \multirow[b]{3}{*}{ No. } & \multirow{2}{*}{\multicolumn{2}{|c|}{$\begin{array}{c}\text { Cradle-to-Gate } \\
\text { Raw material }\end{array}$}} & \multicolumn{5}{|c|}{ Gate-to-Gate } & \multirow{2}{*}{\multicolumn{4}{|c|}{$\begin{array}{c}\text { Gate-to-Grave } \\
\text { Products }\end{array}$}} \\
\hline & & & \multirow{2}{*}{ 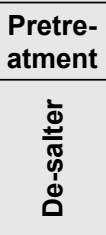 } & \multicolumn{2}{|c|}{ Distillations } & \multicolumn{2}{|c|}{ Enhancers } & & & & \\
\hline & $\begin{array}{l}\overline{0} \\
\frac{0}{0} \\
\bar{\partial}\end{array}$ & 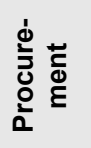 & & 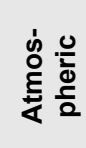 & 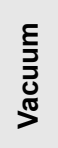 & 紊 & 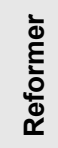 & 志苛 & 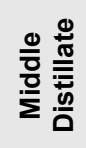 & 离売 & 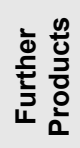 \\
\hline 1 & 0.533 & 1 & -- & -- & -- & -- & -- & 0.286 & 0.250 & 0.286 & 0.320 \\
\hline 2 & 0.967 & 0.5 & 0.667 & 0.767 & 0.6 & 0.667 & 0.7 & 0.867 & 0.967 & 1 & 0.767 \\
\hline
\end{tabular}

\section{Tab. 13: Normalized weighted sum values}

\begin{tabular}{|c|c|c|c|c|c|c|c|c|c|c|c|}
\hline \multirow{3}{*}{ No. } & \multirow{2}{*}{\multicolumn{2}{|c|}{$\begin{array}{c}\text { Cradle-to-Gate } \\
\text { Raw material }\end{array}$}} & \multicolumn{5}{|c|}{ Gate-to-Gate } & \multirow{2}{*}{\multicolumn{4}{|c|}{$\begin{array}{c}\text { Gate-to-Grave } \\
\text { Products }\end{array}$}} \\
\hline & & & \multirow{2}{*}{ 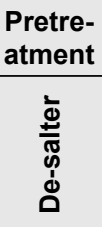 } & \multicolumn{2}{|c|}{ Distillations } & \multicolumn{2}{|c|}{ Enhancers } & & & & \\
\hline & $\begin{array}{l}\overline{0} \\
\frac{0}{0} \\
\frac{\partial}{2}\end{array}$ & 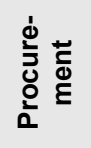 & & 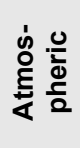 & 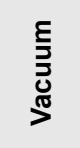 & 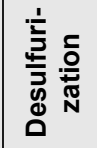 & 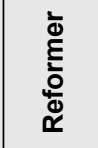 & 奇莺 & 㑒 尊 & 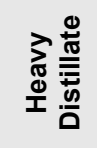 & 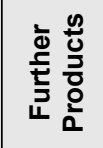 \\
\hline 1 & 0.0053 & 0.010 & -- & -- & -- & -- & -- & 0.0032 & 0.0028 & 0.0032 & 0.0036 \\
\hline 2 & 0.0077 & 0.004 & 0.0052 & 0.006 & 0.005 & 0.0052 & 0.0055 & 0.0077 & 0.0086 & 0.0089 & 0.0068 \\
\hline
\end{tabular}

\section{Tab. 14: Normalized weighted multiplied values}

\begin{tabular}{|c|c|c|c|c|c|c|c|c|c|c|c|}
\hline \multirow[b]{3}{*}{ No. } & \multirow{2}{*}{\multicolumn{2}{|c|}{$\begin{array}{c}\text { Cradle-to-Gate } \\
\text { Raw material }\end{array}$}} & \multicolumn{5}{|c|}{ Gate-to-Gate } & \multirow{2}{*}{\multicolumn{4}{|c|}{$\begin{array}{c}\text { Gate-to-Grave } \\
\text { Products }\end{array}$}} \\
\hline & & & \multirow{2}{*}{ 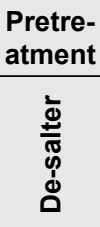 } & \multicolumn{2}{|c|}{ Distillations } & \multicolumn{2}{|c|}{ Enhancers } & & & & \\
\hline & $\begin{array}{l}\overline{0} \\
\frac{0}{0} \\
\bar{\partial} \\
\text { ù }\end{array}$ & 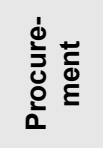 & & 号 & 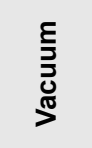 & 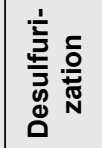 & 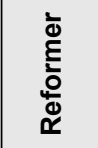 & 莺竞 & 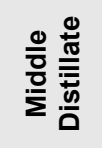 & 恋 & 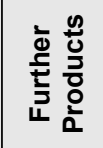 \\
\hline 1 & 0.4938 & 0.5000 & -- & -- & -- & -- & -- & 0.4863 & 0.4848 & 0.4863 & 0.4875 \\
\hline 2 & 0.4997 & 0.4945 & 0.4968 & 0.4979 & 0.496 & 0.4968 & 0.4972 & 0.4987 & 0.4997 & 0.5000 & 0.4976 \\
\hline
\end{tabular}




\begin{tabular}{|c|c|c|c|c|c|c|c|c|c|c|c|}
\hline & \multicolumn{11}{|c|}{ Life-Cycle Phases } \\
\hline & \multicolumn{2}{|c|}{ Raw Material } & \multirow{2}{*}{ 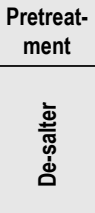 } & \multicolumn{2}{|c|}{ Distillations } & \multicolumn{2}{|c|}{ Enhancers } & \multicolumn{4}{|c|}{ Products } \\
\hline & $\begin{array}{l}\overline{0} \\
\text { oñ } \\
\text { ㄴํㄴ }\end{array}$ & 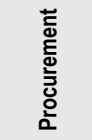 & & 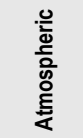 & 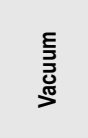 & $\begin{array}{l}\text { 市 } \\
\frac{5}{5} \\
\text { 음 }\end{array}$ & 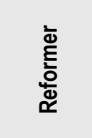 & 蒿恋 & 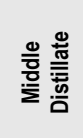 & 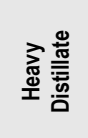 & 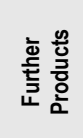 \\
\hline & $\mathrm{A} 1$ & A2 & A3 & A4 & A5 & A6 & A7 & A8 & A9 & $\mathrm{A} 10$ & A11 \\
\hline Scenario1 & 0.4013 & 0.4132 & & & & & & & & & \\
\hline Scenario2 & & & 0.42423 & 0.42404 & 0.42277 & 0.42492 & 0.42483 & & & & \\
\hline Scenario3 & & & & & & & & 0.40806 & 0.40669 & 0.40965 & 0.41340 \\
\hline Primary Ranking & 2 & 1 & 3 & 4 & 5 & 1 & 2 & 3 & 4 & 2 & 1 \\
\hline Participation & \multicolumn{2}{|c|}{0.90099} & \multicolumn{5}{|c|}{0.96039} & \multicolumn{4}{|c|}{0.79207} \\
\hline Secondary Weight & 0.36159 & 0.37237 & 0.40743 & 0.40724 & 0.40602 & 0.40809 & 0.40800 & 0.32322 & 0.32213 & 0.32447 & 0.32744 \\
\hline Normal Weight & 0.08888 & 0.09153 & 0.10015 & 0.10010 & 0.09980 & 0.10031 & 0.10029 & 0.07945 & 0.07918 & 0.07976 & 0.08049 \\
\hline Secondary Ranking & 7 & 6 & 3 & 4 & 5 & 1 & 2 & 10 & 11 & 9 & 8 \\
\hline
\end{tabular}

Source: own

Normalized weighted multiplied values are calculated for every sustainability factor as well (Tab. 14).

Finally, according to the mentioned calculation chain, scenarios and alternatives are primarily and finally weighted and ranked as is illustrated in Tab. 15.

Based on the outputs, poverty, safety, and health are important indicators for the social sustainability, respectively. Moreover, Atmosphere, natural resource, and water, respectively, are the most prominent indicators in the environmental category. Finally, performance of the economy, financial matters, and consumption of energy are three pivotal economic indicators for the case refinery.

Also, desulfurization and reformer which are the most expensive units with the highest influence on the sustainability parameters, and play a prominent role in enhancing the quality of the refinery products, are at the top in the final ranking. Besides, based on the outcome the most important scenario is the Gate-to-Gate or main refinery phase in the life-cycle of the PRI projects. Second to that, comes the Cradle-
to-Gate phase which covers the processes from crude oil excavation, transportation, and procurement services.

\section{Conclusions}

Sustainability evaluation frameworks are widely developed in the industry (building sustainability standards, sustainability reports, etc.) as well as in the academia and research (as is presented in the literature review section). Having said that, a comprehensive integrated framework covering various decision making levels would have a great influence and would be a useful tool for managers in their decision making process.

This study is a part of a chain of studies in the sustainability assessment of petroleum refineries. A sustainability framework based on the indicators and quantitative factors was developed earlier by the authors. In this study the said sustainability framework is applied to PRI projects' life-cycle model. Furthermore, this multistep process is tested for a real refinery case study.

PRI projects are mega-projects and can be categorized and modeled with a life-cycle 
viewpoint in many ways. However, in this study, modeling the project is developed in a concept mapping session so that the model is suitable for sustainability assessment purposes. Afterward, a highly experienced group of experts have detailed the connectivity of the sustainability framework and the PRI life-cycle model during a focus group session. Finally, a real refinery is taken through this methodology via a new scenario based technique.

PRI projects have large impacts on various sustainability attributes in different phases. A comprehensive assessment of these impacts for project's life-cycle is of great importance. The proposed methodology has created the required framework for such assessment by using Concept Mapping, Focus Group, and Multi-Attribute Decision Making Techniques. As an example, the proposed methodology has been applied to a real refinery case. Based on the results, the most important phase in the petroleum refinery life-cycle with regards to all three pillars of sustainability is found to be the operation phase. As such, implementation of various operation phase sustainability aspects should be the first priority of stakeholders during design, procurement, construction, and operation phases. To ensure the continued sustainable performance of the refinery, periodic monitoring and control of the sustainable operation of the refinery are warranted.

\section{References}

Ahmad, W. N. K. W., Rezaei, J., de Brito, M. P., \& Tavasszy, L. A. (2016). The influence of external factors on supply chain sustainability goals of the oil and gas industry. Resources Policy, 49, 302-314. https://doi.org/10.1016/j. resourpol.2016.06.006.

Angelis-Dimakis, A., Arampatzis, G., \& Assimacopoulos, D. (2012). Monitoring the sustainability of the Greek energy system. Energy for Sustainable Development, 16(1), 5156. https://doi.org/10.1016/j.esd.2011.10.003.

Ba-Shammakh, M. S. (2010). Generalized mathematical model for $\mathrm{SO} 2$ reduction in an oil refinery based on arabian light crude oil. Energy \& Fuels, 24(6), 3526-3533. https://doi. org/10.1021/ef1001869.

Brown, L. R. (1982). Building a sustainable society. Society, 19(2), 75-85. https://doi. org/10.1007/BF02712913.

Carson, R. (1962). Silent Spring. New York: Houghton Mifflin Company. de Castro Hilsdorf, W., de Mattos, C. A., \& de Campos Maciel, L. O. (2017). Principles of sustainability and practices in the heavy-duty vehicle industry: A study of multiple cases. Journal of Cleaner Production, 141, 1231-1239. https://doi.org/10.1016/j.jclepro.2016.09.186.

Felix, M., \& Gheewala, S. H. (2012). Environmental assessment of electricity production in Tanzania. Energy for Sustainable Development, 16(4), 439-447. https://doi. org/10.1016/j.esd.2012.07.006.

Ferreira, G., López-Sabirón, A. M., Aranda, J., Mainar-Toledo, M. D., \& Aranda-Usón, A. (2015). Environmental analysis for identifying challenges to recover used reinforced refractories in industrial furnaces. Journal of Cleaner Production, 88, 242-253. https://doi. org/10.1016/j.jclepro.2014.04.087.

Ferreira, F. A. F., Jalali, M. S., Zavadskas, E. K., Meidute-Kavaliauskiene, I. (2017). Assessing Payment Instrument Alternatives Using Cognitive Mapping and the Choquet Integral. Transformations in Business and Economics, 16(2(41)), 170-188.

Filipović, S., \& Golušin, M. (2015). Environmental taxation policy in the EUnew methodology approach. Journal of Cleaner Production, 88, 308-317. https://doi. org/10.1016/j.jclepro.2014.03.002.

George, R. A., Siti-Nabiha, A. K., Jalaludin, D., \& Abdalla, Y. A. (2016). Barriers to and enablers of sustainability integration in the performance management systems of an oil and gas company. Journal of Cleaner Production, 136, 197-212. https://doi.org/10.1016/j.jclepro.2016.01.097.

Hadidi, L. A., AlDosary, A. S., Al-Matar, A. K., \& Mudallah, O. A. (2016). An optimization model to improve gas emission mitigation in oil refineries. Journal of Cleaner Production, 118, 29-36. https://doi.org/10.1016/j. jclepro.2016.01.033.

Halaby, A., Ghoneim, W., \& Helal, A. (2017). Sensitivity analysis and comparative studies for energy sustainability in sewage treatment. Sustainable Energy Technologies and Assessments, 19, 42-50. https://doi. org/10.1016/j.seta.2016.11.004.

Hall, R. P. (2006). Understanding and applying the concept of sustainable development to transportation planning and decision-making in the US. Doctoral dissertation, Massachusetts Institute of Technology.

Hasheminasab, H., Gholipour, Y., Kharrazi, M., \& Streimikiene, D. (2018). A novel Metric 
of Sustainability for petroleum refinery projects. Journal of Cleaner Production, 171, 1215-1224. https://doi.org/10.1016/j.jclepro.2017.09.223.

Hashemkhani Zolfani, S., Maknoon, R., \& Zavadskas, E. K. (2016). Multiple attribute decision making (MADM) based scenarios. International Journal of Strategic Property Management, 20(1), 101-111. https://doi.org/10 .3846/1648715X.2015.1132487.

Heravi, G., Fathi, M., \& Faeghi, S. (2015). Evaluation of sustainability indicators of industrial buildings focused on petrochemical projects. Journal of Cleaner Production, 109, 92-107. https://doi.org/10.1016/j. jclepro.2015.06.133.

Hiremath, R. B., Balachandra, P., Kumar, B., Bansode, S. S., \& Murali, J. (2013). Indicatorbased urban sustainability-A review. Energy for sustainable development, 17(6), 555-563. https://doi.org/10.1016/j.esd.2013.08.004.

Holmgren, K., \& Sternhufvud, C. (2008). CO2-emission reduction costs for petroleum refineries in Sweden. Journal of Cleaner Production, 16(3), 385-394. https://doi. org/10.1016/j.jclepro.2006.11.008.

Jovanovic, J., Jovanovic, M., Jovanovic, A., \& Marinovic, V. (2010). Introduction of cleaner production in the tank farm of the Pancevo Oil Refinery, Serbia. Journal of Cleaner Production, 18(8), 791-798. https://doi.org/10.1016/j. jclepro.2010.01.002.

Mahmoud, A., \& Shuhaimi, M. (2013). Systematic methodology for optimal enterprise network design between bio-refinery and petroleum refinery for the production of transportation fuels. Energy, 59, 224-232. https://doi.org/10.1016/j.energy.2013.06.026.

Missimer, M., Robèrt, K. H., \& Broman, G. (2017). A strategic approach to social sustainability-Part 1: exploring the social system. Journal of Cleaner Production, 140, 32-41. https://doi.org/10.1016/j. jclepro.2016.03.170.

Missimer, M., Robèrt, K. H., \& Broman, G. (2017). A strategic approach to social sustainability-Part 2: a principle-based definition. Journal of Cleaner Production, 140, 42-52. https://doi.org/10.1016/j. jclepro.2016.04.059.

Musango, J. K., \& Brent, A. C. (2011). A conceptual framework for energy technology sustainability assessment. Energy for Sustainable Development, 15(1), 84-91. https://doi.org/10.1016/j.esd.2010.10.005.
Neelis, M. (2008). Energy Efficiency Improvement and Cost Saving Opportunities for the Petrochemical Industry-An ENERGY STAR (R) Guide for Energy and Plant Managers.

Ooba, M., Hayashi, K., Fujii, M., Fujita, T., Machimura, T., \& Matsui, T. (2015). A longterm assessment of ecological-economic sustainability of woody biomass production in Japan. Journal of Cleaner Production, 88, 318-325. https://doi.org/10.1016/j. jclepro.2014.09.072.

Papajohn, D., Brinker, C., \& El Asmar, M. (2016). MARS: Metaframework for assessing ratings of sustainability for buildings and infrastructure. Journal of Management in Engineering, 33(1), https://doi.org/10.1061/ (ASCE)ME.1943-5479.0000478.

Shen, L. Y., Ochoa, J. J., Shah, M. N., \& Zhang, X. (2011). The application of urban sustainability indicators-A comparison between various practices. Habitat International, 35(1), 17-29. https://doi.org/10.1016/j. habitatint.2010.03.006.

Shen, L., Wu, Y., \& Zhang, X. (2010). Key assessment indicators for the sustainability of infrastructure projects. Journal of Construction Engineering and Management, 137(6), 441-451. https://doi.org/10.1061/(ASCE) Co.1943-7862.0000315.

Shortall, R., Davidsdottir, B., \& Axelsson, G. (2015). Development of a sustainability assessment framework for geothermal energy projects. Energy for Sustainable Development, 27, 28-45. https://doi.org/10.1016/j.esd.2015.02.004.

Sierra, L. A., Pellicer, E., \& Yepes, V. (2015). Social sustainability in the lifecycle of chilean public infrastructure. Journal of Construction Engineering and Management, 142(5). https://doi.org/10.1061/(ASCE)CO.19437862.0001099.

Silajdžić, I., Kurtagić, S. M., \& Vučijak, B. (2015). Green entrepreneurship in transition economies: a case study of Bosnia and Herzegovina. Journal of Cleaner Production, 88, 376-384. https://doi.org/10.1016/j. jclepro.2014.07.004.

Silvestre, B. S., \& Gimenes, F. A. P. (2017). A sustainability paradox? Sustainable operations in the offshore oil and gas industry: The case of Petrobras. Journal of Cleaner Production, 142, 360-370. https://doi. org/10.1016/j.jclepro.2016.07.215.

Stamford, L., \& Azapagic, A. (2014). Life cycle sustainability assessment of UK electricity 
scenarios to 2070. Energy for Sustainable Development, 23, 194-211. https://doi. org/10.1016/j.esd.2014.09.008.

Stelzer, B., Meyer-Brötz, F., Schiebel, E., \& Brecht, L. (2015). Combining the scenario technique with bibliometrics for technology foresight: The case of personalized medicine. Technological Forecasting and Social Change, 98, 137-156. https://doi.org/10.1016/j. techfore.2015.06.008.

Stjepcevic, J., \& Siksnelyte, I. (2017). Corporate Social Responsibility in Energy Sector. Transformations in Business and Economics 16(1(40)), 21-34.

Streeten, P. (2001). Globalisation: Threat or opportunity? (pp. 30). Copenhagen: Copenhagen Business School Press.

Wang, Y., Shi, X., Sun, J., \& Qian, W. (2014). A grey interval relational degree-based dynamic multiattribute decision making method and its application in investment decision making. Mathematical Problems in Engineering. https://doi.org/10.1155/2014/607016.

Yao, H., Shen, L., Tan, Y., \& Hao, J. (2011). Simulating the impacts of policy scenarios on the sustainability performance of infrastructure projects. Automation in Construction, 20(8), 1060-1069. https://doi.org/10.1016/j. autcon.2011.04.007.

Zavadskas, E. K., Turskis, Z., \& Kildienè, S. (2014). State of art surveys of overviews on MCDM/MADM methods. Technological and economic development of economy, 20(1), 165-179. https://doi.org/10.3846/20294913.20 14.892037.

Prof. Yaghob Gholipour, PhD University of Tehran College of Engineering Engineering Optimization Research $\mathrm{Gr}$. Iran ygholipour@ut.ac.ir

Hamidreza Hasheminasab University of Tehran College of Engineering School of Civil Engineering Iran hasheminasab@ut.ac.ir

Mohammad Kharrazi, PhD Amir Kabir University of Technology Office of Sustainable Development Iran mkharrazi@hotmail.com

Justas Streimikis Lithuanian Institute of Agrarian Economics Division of Farms and Enterprises Economics Lithuania Justas.streimikis@gmail.com 


\title{
Abstract
}

\section{SUSTAINABILITY CRITERIA ASSESSMENT FOR LIFE-CYCLE PHASES OF PETROLEUM REFINERY PROJECTS BY MADM TECHNIQUE}

\author{
Yaghob Gholipour, Hamidreza Hasheminasab, Mohammad Kharrazi, \\ Justas Streimikis
}

PRI projects have large impacts on various sustainability attributes in different phases. A comprehensive assessment of these impacts for project's life-cycle is of great importance. The proposed methodology has created the required framework for such assessment by using Concept Mapping, Focus Group, and Multi-Attribute Decision Making Techniques.

Petroleum refinery industry (PRI) is comprised of a chain of expertise and engineering disciplines and is also connected to various other industries. Consequently, apart from its physical products that fulfill the fuel needs of the society, PRI has a great contribution to economic development and to the prosperity of different businesses. On the negative side, however, PRI is one of the primary contributors to global warming and other environmental issues and could have some unwelcome effects on the society as well. Hence, development of these projects should be done with careful assessment of their life-long impacts from a sustainability viewpoint.

In this study, a sustainability framework for PRI projects, which was developed and presented in a previous publication, is used in a multiphase methodology to assess the relationship between lifecycle phases and sub-phases of a petroleum refinery on one hand, and sustainability indicators and factors on the other hand. As a result of this study, critical sustainability factors can be highlighted, to help managers and industry experts consider long-term sustainability consequences in their decision making process. The proposed methodology has been applied to a real petroleum refinery project, using an MADM-based scenario approach, to assess the correlation between sustainability factors and various life-cycle phases and sub-phases of the refinery.

Based on the results, the most important phase in the petroleum refinery life-cycle with regards to all three pillars of sustainability is found to be the operation phase. As such, implementation of various operation phase sustainability aspects should be the first priority of stakeholders during design, procurement, construction, and operation phases.

Key Words: Sustainability assessment, petroleum refinery, life-cycle modeling, Multi-Criteria Decision Making, MADM.

JEL Classification: Q01, Q35, Q51.

DOI: 10.15240/tul/001/2018-3-005 